\section{Statistical commentary}

\author{
Robert W Platt
}

Editors note: Two recent events promoted me to ask Robert Platt, a biostatistician, to contribute occasional pieces to the journal under the heading, statistical commentary. The first is the appearance of papers using statistical procedures that may only be familiar to some readers. My hope is that this column will help explain these procedures in simple terms so that the scientific papers are more generally understood. The second is a recent report in the New England fournal of Medicine describing an apparent increase in crashes involving cellphones. This received wide coverage in the media; equally interesting were the bizarre responses of the cellphone industry to this report. Although not a problem that directly affects children or adolescents, if the results are valid the indirect effects could involve young people. (Besides, I have a prejudice against the use of cellphones in public and especially while driving!)

\section{Statistical commentary: cellular telephones and motor vehicle accidents}

Cellular and other portable telephones are one of the fastest growing parts of the telecommunications industry. Portable communications allow for large improvements in productivity and safety for many people. However, they are also potentially a cause of significant impairment of driving performance when used to make or receive calls while driving.

The recent study by Redelmeier and Tibshirani examines the possible link between cellular telephone calls and motor vehicle accidents. ${ }^{1}$ They report a substantial increased risk of motor vehicle accidents when a cellular telephone was being used (relative risk 4.3, $95 \%$ confidence interval 3.0 to 6.5 ). The risk persisted across various characteristics of the driver and situation (education level, age, driving and cellular phone experience, type of telephone (hand-held or hands-free)). This risk is substantial, and is approximately the same as that associated with driving with a blood alcohol level at the legal limit. (The study also found that several drivers used cellular telephones to contact authorities and emergency personnel after the accident.)

The design is an interesting and useful one - a case-crossover design. ${ }^{2}$ One difficulty with any case-control study is that the researcher must enlist controls from a population similar to the cases. If the cases and controls differ in some way unrelated to the outcome or the exposure a number of possible biases may arise. To get around this, the case-crossover design uses the cases themselves at an earlier point in time when they were at risk for the event but did not experience it. In this example, the same people, at the same time of day, are used as controls. This effectively matches for all fixed characteristics of the driver. This design requires, however, that possible confounding factors and the exposure itself do not vary over time.

There are several points to consider in this study. First, the authors note that there are potential confounding factors that occur due to temporary differences in driver characteristics or circumstances (other impairments, weather conditions) that may affect the results but it is unlikely that these factors would account for the entire risk difference. Second, the study participants differ in some ways from typical cellular users: most importantly in terms of usage. The participants were higher than average users of cellular telephone, and were those who agreed to participate. These factors suggest that the study population may be representative of people who are more experienced with telephones and also, perhaps, safer drivers (because one suggested reason for nonparticipation is fear of liability). Third, each accident time is compared with only one point in time previous to the accident. Better results might be achieved with the averaging of multiple control time periods. Fourth, the study only investigated reported accidents and did not differentiate between types or causes other than to exclude collisions involving injury, criminal activity, and transport of dangerous goods. This point is not likely to induce bias but reduces the generalizability of the results to injury accidents or those unlikely to be reported. Finally, extreme caution must be exercised in case-crossover studies to ensure that the exposure (cellular telephone calling while driving) distribution is stable over time. There is no reason to assume in advance that this was so, but Redelmeier and Tibshirani adequately account for the possibility with a pilot study of people who were definitely driving during the control period.

These are all minor criticisms, none of which is likely to explain such a large relative risk. Thus this is a valuable study - one that raises important questions about driving and motor vehicle accidents. The evidence on hands-free telephones suggests that mental distraction and not decreased physical dexterity is the key factor in the increased risk. This implies that other driving distractions such as car radios, eating, and even discussions with passengers may be other possible risk factors. It also suggests many future studies on carphone use, in particular, to determine if different types of conversations result in different risks due to different distraction levels. Clearly, a more detailed examination of the differences between hand-held and hands-free phones is needed. An area requiring further study is the type and cause of the collisions in question. A larger study that included motor vehicle injuries, and adjusted for driver responsibility,

\section{Correspondence to: $\mathrm{Dr} \mathbf{R} \mathrm{W}$ Platt, McGill/Montreal Research Institute, 2300 Tupper, Montreal, PQ H3H IP3, Canada. \\ Pediatrics, Epidemiol- ogy, and Biostatistics,}


would clarify the public health implications of the association found in this report.

It may be premature to consider changes in the legal approach to cellphone use in cars (although some countries require that the car be pulled over and stopped while doing so; others ban car telephone use outright, while others ban only hand-held phones). At the very least this study should suggest to cellular telephone service providers and users that unnecessary use of these devices while driving should be discouraged. Although it is difficult to imagine a situation where the use of a telephone while driving is necessary, should this arise extreme caution is needed.

1 Redelmeier DA, Tibshirani RI. Association between cellula telephone calls and motor vehicle collisions. $N$ Engl $\mathcal{F} M e \dot{d}$ 1997; 336: 45-8.

2 Maclure M. The case-crossover design: a method for studyin transient effects on the risk of acute events. $A m \Phi$ Epidemiol 1991; 133: 144-53.

International Society for Child and Adolescent Injury Prevention

We invite you to join the International Society for Child and Adolescent Injury Prevention (ISCAIP). ISCAIP was created in 1993 for injury professionals around the world. The goal of ISCAIP is to reduce the number and severity of injuries to children and adolescents through international collaboration.

\section{Membership fee}

The annual membership fee for ISCAIP, including a subscription to Injury Prevention, is: $£ 80$ for individuals

$£ 125$ for non-profit or charity institutions

$£ 250$ for corporate institutions

If you would like to receive a brochure describing ISCAIP in greater detail, please write to the address below.

\section{How to join}

Please complete this form and return it to ISCAIP, c/o CAPT, 18-20 Farringdon Lane, London EC1R 3AU, UK

Name (Mr/Mrs/Ms/Miss/Dr):

Title/position:

Institution:

Address (plus postal/zip code)

Telephone/fax/e-mail

Type of membership (ring one)

Individual/non-profit charity/corporate

Visa/Mastercard number ${ }^{\star}$

Expiry date

Name as it appears on the card

Card billing address (if different from above)

Amount to be charged

Signature of cardholder

*When paying by credit card, the account will be charged in pounds sterling and converted accordingly (we much prefer this method of payment). 